\title{
The Prevalence of Early Childhood Caries (ECC) and Its Associated Risk Factors Among Immigrant Tibetan Pre-School Children in Bylakuppe, Mysore, India
}

\author{
Madhusudan Kushalappa. Kaikure ${ }^{1, ~}{ }^{*}$, Ann Thomas ${ }^{1}$, Sowmya B. Shetty ${ }^{1}$, Tony Jose ${ }^{2}$, \\ Raghavendra Pidamale ${ }^{1}$, Savitha Lakshmi Kaikure ${ }^{3}$ \\ ${ }^{1}$ Dept.of pedodontics and preventive dentistry, A J Institute of dental sciences, Mangaluru, India \\ ${ }^{2}$ Dept.of pedodontics and preventive dentistry, Indira Gandhi dental college, Cochin, India \\ ${ }^{3}$ Dept. of oral and maxillofacial pathology, A J Institute of dental sciences, Mangaluru, India \\ Email address: \\ dr.mkkaikure@gmail.com (M. K. Kaikure),dr_mkkaikure@yahoo.com (M. K. Kaikure), annkotts@hotmail.com (A. Thomas), \\ sowmyab@live.com (S. B. Shetty),dr.tonyjose@live.com (T. Jose),drpidamale@yahoo.com (R. Pidamale), \\ savimadsk@yahoo.com (S. L. Kaikure)
}

\section{To cite this article:}

Madhusudan Kushalappa. Kaikure, Ann Thomas, Sowmya B. Shetty, Tony Jose, Raghavendra Pidamale, Savitha Lakshmi Kaikure. The Prevalence of Early Childhood Caries (ECC) and its Associated Risk Factors Among Immigrant Tibetan Pre-School Children in Bylakuppe, Mysore, India. Science Journal of Public Health. Vol. 3, No. 3, 2015, pp. 384-390. doi: 10.11648/j.sjph.20150303.23

\begin{abstract}
Objectives: This study estimated the prevalence of early childhood caries (ECC) and related risk factors in a population of immigrant Tibetan pre-school children in Bylakuppe of Mysore District; India. Methodology: A cross sectional study was conducted in 500 pre-school children between 10 to 72 months of age in and around Bylakuppe of Mysore district; India; using II stage stratified random sampling. The caries experience was recorded using defs and the deft index. Information regarding risk factors for caries was obtained through a structured questionnaire given to mothers/caretakers. Statistical data analysis: The data was statistically analyzed by using "chi-square" test and "ANOVA" test. Results: The prevalence of ECC in the study sample was significantly high. It was $92.2 \%$ with the mean defs score of 10.27 and the mean deft score of 6.15 $(\mathrm{p}<0.001 \mathrm{vhs})$.Higher prevalence of ECC was found, in children with bottle feeding, addition of sugar to bottle content and to regular food, in-between meal snacking habits and increase intake of sweets like chocolate, candies and toffees, lesser frequency of brushing and child brushing unassisted/unsupervised. Reports of regular visits to the dentist were almost absent in the study population. Conclusion: This study demonstrates the high prevalence of ECC in the study population. Development of strategies and protocols; to prevent and manage ECC, based on the risk factors identified for these population, is essential to curb this oral health problem.
\end{abstract}

Keywords: ECC, Caries Experience, Feeding and Dietary Practices, Oral Hygiene Practices, Regular Dental Visits, Socio-Economic Status

\section{Introduction}

Early Childhood Caries (ECC) has been a major health problem over many years and still continues today, affecting the physical growth and development, as well as the social adaptation of young children. It is a rampant, chronic, transmissible, infectious disease, which can rapidly destroy the primary dentition of toddlers and young children (Filstrup SL et al., 2003), with a complex and multi-factorial etiology; which includes excessive bottle feeding with sugar containing liquids, "at will" breast feeding and/or falling asleep while feeding and nursing beyond the recommended age for weaning (Man Qin et al., 2008). It associated with other health problems, range from local pain, infections, abscesses, leading to difficulty in chewing, malnutrition, gastrointestinal disorders, difficulty in sleeping (Low W et al., 1999); and also impairing the quality of life and low selfesteem (Tracy L et al., 2007). Furthermore, children withECC are at a greater risk for increased caries experience along the continuum of childhood (Kaste LM et al., 1992; Li 
Y and Wang W 2002).

American Academy of Pediatric Dentistry (AAPD) in 2003 defined ECC as the presence of one or more decayed (non-cavitated or Cavitated), missing (due to caries), or filled tooth surface in any primary tooth in a child 71 months of age or younger. The current definition of ECC is the presence of at least one primary tooth affected by caries in children under 6 yrs of age (Robert JS et al., 2005).It encompasses all previous terminologies, namely baby bottle tooth decay, baby bottle syndrome, labial caries, nursing caries, nursing bottle caries, nursing bottle syndrome, rampant caries etc; including sub groups with minimal or isolated decay extending to those with rampant manifestations (Robert JS et al., 2007). Prevalence of ECC also varies widely with several factors like race, culture and ethnicity; socioeconomic status, life style, dietary pattern and oral hygiene practices (Murray JJ 1996). Culture is characterized by different indigenous beliefs that directly impact on child-rearing practices, the nature of family roles and health beliefs (Laws MB 2001). Some cultures place little value on primary teeth, Native Americans consider ECC as a normal childhood disease affecting children (Man Wai Ng 2003).

Numerous studies have been conducted worldwide to find the prevalence of ECC. Data on preschool children are sparse and often lack adequacy in sample size and representativeness; because children at these ages are not readily accessible for examination and are difficult to examine thoroughly (Vadiakas G 2008). A comprehensive literature review of the prevalence of ECC found it highest in South East Asia (36 -85 \%) and to be low in developed countries like USA and England $11-53 \%$ and $6.8-12 \%$ respectively (Vivek DK 2010). There is paucity of studies on ECC prevalence and its associated risk factors; in the immigrant Tibetan population worldwide.

Background: Invasion of Tibet by China in 1950 led to an exodus of Tibetans to India; who have immigrated and settled in small disparate communities in different parts of the country. These immigrant Tibetans continue to practice their traditional lifestyles, beliefs, customs and dietary habits; with only negligible changes made to suit the local environment (Robertson J et al., 1989). A marked difference in lifestyle, culture, beliefs and practices are visible in the immigrant Tibetan population in Bylakuppe of Mysore district: India.

Hence, the aim of this study is to assess the prevalence of ECC and the associated risk factors among the immigrant Tibetan children below 6 years of age residing in Bylakuppe of Mysore district; India.

\section{Methodology}

This cross sectional study was conducted in the immigrant Tibetan pre-school children $(\mathrm{N}=500)$ in Bylakuppe of Mysore district; India, to assess the prevalence of ECC and its associated risk factors.

Sampling procedure: The study population consisted of 500 immigrant Tibetan children (266 males and 234 females) between 10 to 72 months of age. All the immigrant Tibetan children were selected from the ten pre-schools; located at Tibetan settlements in Bylakuppe of Mysore district; India.

Inclusion criteria:The study sample was selected based on the following criteria:

- Children below 6 years of age only.

- Children with only primary dentition.

- Children with a class I physical status according to American Society of Anesthesiologists (ASA).

Approval for the study was obtained from the respective authorities. The parents of the study participants and the teachers/caretakers; were given clear explanations about the objective of the study and written consent was obtained; to collect information and to carry out clinical examination of the children.

Dental examination procedure: The dental examination was performed by one qualified dentist with the aid of a dental diagnostic set (plane mouth mirror, CPI probe and tweezer). Personal protective barriers like gloves and masks were used. Chemical sterilizing solution was used for instrument sterilization. CPI probe was used to confirm visual evidence of caries on the occlusal, buccal and lingual surfaces. Children were examined seated on an upright chair in adequate natural light and the data was recorded by a separate recorder. The caries experience was recorded using defs and the deft index.

Data collection: A questionnaire was developed in English; containing 23 questions to collect the relevant data of the children's demographic details (8 questions), socioeconomic status (2 questions), oral hygiene habits (6 questions); dietary habits (5 questions) and dental visits to the dentist (2 questions). Part of the questionnaire on dietary habits, was worded differently for children below 3 years and above 3 years to elicit the correct details. The children's parents were instructed to complete the questionnaires to the best of their understanding.

Data analysis: The data was statistically analyzed by using "chi-square" test and "ANOVA" test.

\section{Result}

The study sample consisted of 500 Immigrant Tibetan children, of which 266 were males and 234 were females with age ranged from 10 to 72 months. (Mean age= $3.896 \mathrm{yrs} / 45$ months). It was very highly statistically significant $(\mathrm{p}<0.001)$ (Table. 1$)$.

The prevalence of ECC was $92.2 \%$; with the mean defs score of 10.2 and the mean deft score of 6.15 ( $p<0.001 \mathrm{vhs})$. According to the age distribution, the prevalence of ECC among the children below 36 months was $82.6 \%$; children aged between $36-47$ months was $97 \%$; children between 48 59 months was $96.1 \%$ and children between $60-72$ months was $91.4 \%$ (Table. 2 ).

In this study, $89.2 \%$ of the Immigrant Tibetans have reported to be practicing the oral hygiene habit of tooth brushing. The majority of the children reported to be using tooth brush and toothpaste, and the prevalence of ECC in these children was $90.1 \%$ and only $1.3 \%$ of the children 
reported of using finger. No statistically significant difference was found in the ECC prevalence and the different aids used for tooth brushing. The prevalence of ECC decreased when the frequency of tooth brushing was greater. It was found statistically significant $(\mathrm{p}=0.012)$. In this study, only $4.2 \%$ of the immigrant Tibetan children assisted by the parent in tooth brushing. Majority of the children reported to be brushing alone. The prevalence of ECC was found higher among those who brushed unassisted/unsupervised than those who had brushed under parental supervision/ assistance. It was highly significant $(\mathrm{p}=0.006) .50 \%$ of immigrant Tibetan children reported positively of rinsing mouth after every meal. ECC prevalence was found more among those who had not rinsed their mouth after every meal. No statistical difference was found between ECC prevalence and this habit(Table.3).

In this study, majority of the mothers had breast-fed their child. More than $50 \%$ of the children's caretakers did not remember the frequency of feeds during the day and night. Majority of the children weaned from breast feeding at the age of $1 \mathrm{yr}$. There was no statistical significant correlation between ECC experience and breast feeding habit in the population. Bottle feeding practice was very much common among the immigrant Tibetan children (81.4\%); and this was statistically highly significant $(\mathrm{p}<0.001 \mathrm{vhs})$. Only $1.3 \%$ of the immigrant Tibetan sample had reported of still breast feeding after $3 y r s$ of age. The common content of the bottle was cow's milk and infant formula. The prevalence of ECC was found higher in the bottle fed children; and was statistically very highly significant $(\mathrm{p}<0.001 \mathrm{vhs})$. The habit of adding table sugar to the bottle content was found in $80.0 \%$ of the bottle fed sample. The ECC experience was higher in those with this habit and was statistically highly significant ( $p<0.001 \mathrm{vhs}$ ). $76.8 \%$ of the immigrant Tibetan children reported of consuming regular or complementary foods. $88.0 \%$ reported the habit of adding sugar to the food and this habit showed very high significant prevalence of ECC $(<0.001 \mathrm{vhs})$. $67.4 \%$ of the children aged $3 y r s$ and above reported to a frequency of five regular meals per day (table.4).

The habit of in-between-meal snacking was considerably low among the immigrant Tibetan children. $18.8 \%$ Tibetan children reported to the habit of in-between-meal snacking, while $20.4 \%$ did not respond to this question. It shows that, the correlation between ECC and in-between meal snacking habit was very highly significant. $82 \%$ of the immigrant Tibetans children reported to the presence of the habit of eating sweets. ECC prevalence was more among those who had regular sweet intake (chocolates, candies, toffees). There was statistically very high significance $(p<0.001)$ in the correlation between ECC and sweet intake in the population (Table.4).

Only $17 \%$ of the immigrant Tibetan children had been to the dentist. Among those who have not visited dentist; 91\% of the children experienced ECC. It shows high significance (.006hs), when correlated on ECC prevalence and visit to the dentist. $90.6 \%$ of the immigrant Tibetan children had been to the dentist due to tooth related problems such as pain, swelling, trauma etc; and only $9.3 \%$ of the children had visited dentist for regular check up. Among those who had been to the dentist, $67.5 \%$ of the children had received treatment like amalgam restoration and extraction (table.5).

\section{Discussion}

ECC is a virulent form of dental caries that can destroy the primary dentition of toddlers and preschool children. Despite the major advances in the field of caries prevention over the decades, there are reports of early childhood caries in young children are still high (Robert. J and Berkowitz 2003). ECC universally thought to be an endemic, and is severe in socioeconomic, immigrant and indigenous communitieswith ECC prevalence reported to range from $11-75 \%$ (Cunnigham SED et al.,2008).

ECC Prevalence: In this study, the prevalence of ECC in the immigrant Tibetan sample was significantly higher. It was $92.2 \%$ with a mean defs score of 10.27 and deft score of 6.15 and only $7.8 \%$ was caries free (Table No.2). This is similar to the other reports of the immigrant population having higher prevalence of ECC (Ekman A et al., 1981; Tang JMW et al.,1997). No data is available regarding ECC prevalence in the Tibetan populations or in the immigrant Tibetans in India. In children aged between 5-6yrs, 8.6\% were caries free with a mean deft score of 7.04; it was higher to the value reported in the similar population in Dhar; India, $20 \%$ of the children were caries free and the mean deft score was $\operatorname{six}($ Robertson J et al., 1989). The ECC prevalence in the Tibetan population, when compared to the developed and developing countries was higher. In children aged 3yrs, the ECC prevalence in immigrant Tibetans was $97 \%$, and is considerably higher to the value reported in Philippines (Cariono KM et al., 2003)which was $80 \%$; and was more than twice the value reported in Turkey which was 40\%(Namal $\mathrm{N}$ et al., 2005). In the children aged 4yrs, ECC prevalence in immigrant Tibetans was 96.1\%; which considerably more than the values reported in Scotland (Sweeney PC and Gel bier S 1999) and Philippines (Cariono $\mathrm{KM}$ et al.,2003) which was $86 \%$ and $90 \%$ respectively; whereas it is almost twice the value reported in Turkey which was $50 \%$ (Namal $\mathrm{N}$ et al., 2005). In children aged 5yrsECC prevalence in immigrant Tibetans was $91.4 \%$; and is lower than the value reported in Philippines which was $94 \%$ (Cariono KM et al., 2003); higher than the value reported in Turkey which was 69\% (Namal N et al., 2005), and is more than thrice the value reported in Denmark which was $29.1 \%$ (Poulsen S and Scheutz F 1999). Also in the children aged 35 yrs. ECC prevalence in immigrant Tibetans was morethan those reported in Maryland USA, which was $52 \%$ (Clemencia M et al., 2000), and in children aged 6 to 59 months in Korea which was 56.5\% (Jin BH et al., 2003).

Oral Hygiene Practices and ECC Prevalence: Many researchers have reported that the prevalence of regular tooth brushing has a positive impact on dental caries (Dominguez RV et al., 1993; Verrips GH et al., 1993). No such effect was observed in this study; though $89.2 \%$ of the study sample practiced regular tooth brushing habit (Table.3). Similar 
finding was reported in Turkey (Namal $\mathrm{N}$ et al., 2005). Majority of the children $(82 \%)$ in the population used toothbrush and paste; this could be attributed to the increase in media advertisements and ease of availability. No significant difference in ECC prevalence was observed with the different aids used for tooth brushing; which is contradictory to the earlier report (Chan SCL et al., 2002). Most children (68.6\%) have reported to be brushing their teeth unassisted and, or unsupervised; though tooth brushing in this age group is to be carried out under parental supervision or assistance. And like the earlier reports. Prevalence of ECC in this population was very high; since most mothers let children brush their teeth themselves; because of the difficulties and time constraints (Chan SCL et al., 2002; Jose B and King NM 2003; Kuriakose S and Joseph E 1999). There was significant decrease in ECC prevalence with increase in tooth brushing frequency in the population; similar to the previous reports(Jose B and King NM 2003; Rodrigues CS and Sheham A. 2000). Majority of the children $(69.2 \%)$ brushed their teeth only in the morning in both the population.

Feeding and Dietary Habits and ECC Prevalence:

Feeding practices: Breast feeding is a common accepted feeding practice in the immigrant Tibetan population. Majority of the mothers (90.2\%) (Table.4)of the study population had breast fed their children; but was unable to recall the frequency. Majority of the children (43\%) reported to have weaned from breast feeding and to have started bottle feeding by the age of 1yr. No significance in ECC prevalence was found in relation to the breast feeding habit. Systematic review of scientific literature suggests contradictory findings between breastfeeding and development of ECC (Valaitis R, et al., 2000). Majority of the immigrant Tibetan study sample $(81.4 \%)$ reported to have bottle fed their children, and highly significant association was found between bottle feeding practice and ECC prevalence in the study population. Bottle feeding practice beyond the age of 1 year at night-time, was common among these population. Reports suggest a strong association between this variable and development of ECC (Verrips GH et al., 1993).The contents of the bottles were usually milk and infant formula; whereas $67.6 \%$ of the study sample reported positively to the habit of adding table sugar to the bottle content, and strong significance was found between this variable and ECC prevalence. Similar reports are present suggesting this variable as risk factor for ECC development (Chan SCL et al., 2002; Jin BH et al., 2003; Robert JS et al., 2005).

Dietary practices:Majority of the study sample reported to be taking complementary or regular food in the population. $88 \%$ of the immigrant Tibetans reported positively to the habit of adding table sugar or some other sweetener to the food. Significantly higher ECC prevalence was found in association to this variable. The habit of in-between meal snacking was considerably lower (18.8\%) (Table.4).This could be due to the longer school hours and community school meal programs for the immigrant Tibetan children. Highly significant association was found between ECC prevalence and the habit of in-between meal snacking in both the population; similar to the previous reports (Chan SCL et al., 2002; Jin BH et al., 2003; Jose B and King NM 2003). $82 \%$ of the immigrant Tibetan study sample reported positively to the habit of eating candies, toffees, chocolates etc; significantly high ECC prevalence was found in relation to this variable (Table.4). Reports suggest higher caries increment with increased sugar intake, and lesser oral clearance /greater retain ability of sugars (Chan SCL et al., 2002; Jin BH et al., 2003; Jose B and King NM 2003;Rodrigues CS and Sheham A. 2000).

Routine Dental Visits and ECC Prevalence:In this study, only $17.2 \%$ of the study sample had visited the dentist. None had reported to have been to the dentist for a routine dental check up, but rather for specific treatment needs, such as pain, swelling, trauma, etc. Likewise higher ECC Prevalence was found in those children, who had reported, to have visited the dentist (Table.5). Similar reports are also observed in Korea (Jin BH et al., 2003) and china (Man Qin et al.,2008). Regarding the dental treatment, eleven immigrant Tibetan children reported to have undergone, including silver amalgam and temporary restorations, extractions and oral prophylaxis. Evidence suggests that, initiation of routine dental checkups before one year of age, could aid in education and reinforcement of healthy feeding practices and oral hygiene habits in the parents; and also in prevention, early detection and management of incipient carious lesions and before its deleterious effects (Chan SCL et al., 2002; Jin BH et al., 2003).

\section{Conclusion}

This study indicates that ECC is a serious health problem affecting the immigrant Tibetan children in Bylakuppe of Mysore district; India. The prevalence of ECC was very high in this population; greater than the national and worldwide reports.

Assessment of retrospective interview data of the children's oral hygiene practices, feeding/dietary habits and practices, and dental visits revealed the followings:

- Majority of the children in the study population brushed their teeth unassisted/unsupervised by the parent with toothbrush and toothpaste.

- Higher prevalence of ECC was found, with the use of other tooth brushing aids, than the toothbrush and toothpaste; lesser frequency of brushing and the child brushing unassisted/unsupervised by the parent.

- Higher prevalence of ECC was found, with feeding/dietary habits like bottle feeding; addition of sugar to bottle content and to regular food; and increase intake of sweets like chocolate, candies and toffees.

- Reports of regular visits to the dentist were almost absent in the study population. Few who reported to have visited the dentist, had done so, for alleviating dental problems like pain, swelling, trauma etc.

This study demonstrates the high prevalence of the disease in the immigrant Tibetan population; and there is an urgent 
need for treatment and promotion of oral health awareness factors. and management strategies; based on the identified risk

Table 1. Distribution of the population based on sex and mean age.

\begin{tabular}{|c|c|c|c|c|c|c|c|}
\hline \multirow{3}{*}{ Sample } & \multicolumn{4}{|c|}{ Sex Distribution } & \multicolumn{3}{|l|}{ Age } \\
\hline & \multicolumn{2}{|c|}{ Males } & \multicolumn{2}{|c|}{ Females } & \multirow{2}{*}{ Mean } & \multirow{2}{*}{ S.d } & \multirow{2}{*}{ T'value } \\
\hline & $\mathbf{N}$ & $\%$ & $\mathbf{N}$ & $\%$ & & & \\
\hline Tibetan & 266 & 53.2 & 234 & 46.8 & 3.8969 & 1.11823 & $\begin{array}{l}8.80300 \\
\mathrm{P}<0.001 \mathrm{vhs}\end{array}$ \\
\hline
\end{tabular}

Table 2. Prevalence of ECC by age in the population and the mean defs\& deft value.

\begin{tabular}{|c|c|c|c|c|c|c|c|c|c|c|c|c|c|}
\hline \multirow{2}{*}{ Population } & \multirow{2}{*}{ Caries status } & \multicolumn{2}{|c|}{$<36$ months } & \multicolumn{2}{|c|}{ 36-47months } & \multicolumn{2}{|c|}{ 48-59months } & \multicolumn{2}{|c|}{ 60-72months } & \multicolumn{2}{|c|}{ Total sample(500N) } & \multicolumn{2}{|c|}{ Total sample(500N) } \\
\hline & & $\mathbf{n}$ & $\%$ & $\mathbf{n}$ & $\%$ & $\mathbf{n}$ & $\%$ & $\mathbf{N}$ & $\%$ & $\mathbf{N}$ & $\%$ & mean defs & mean deft \\
\hline Tibetan & $\mathrm{ECC}$ & 81 & 82.6 & 105 & 97.0 & 125 & 96.1 & 150 & 91.4 & 461 & 92.2 & 10.2 & 6.15 \\
\hline
\end{tabular}

ECC: $\mathrm{X}^{2}=62.12 ; \mathrm{p}=$ value $<0.001$ (vhs); defs: $\mathrm{F}=9.88 ; \mathrm{p}=<0.001$ (vhs); deft: $\mathrm{F}=13.2 ; \mathrm{p}==<0.001$ (vhs)

Table 3. Oral hygiene practices and ECC prevalence.

\begin{tabular}{|c|c|c|c|c|c|}
\hline \multirow{2}{*}{ Oral hygiene practices } & \multirow{2}{*}{ Variables } & \multicolumn{4}{|c|}{ TIBETAN } \\
\hline & & $\mathbf{N}$ & $\mathbf{n}$ & $\%$ & $\mathbf{P}$ \\
\hline Tooth brushing & Yes & 446 & 428 & 95.9 & $<0.748$ (ns) \\
\hline \multirow{5}{*}{ Aids used for tooth brushing } & Only Brush & 70 & 65 & 92.8 & \multirow{5}{*}{$\mathrm{p}=0.841(\mathrm{~ns})$} \\
\hline & Only finger & 6 & 6 & 100.0 & \\
\hline & Brush and Tooth Paste & 366 & 330 & 90.1 & \\
\hline & Finger and tooth paste & 1 & 1 & 100.0 & \\
\hline & Any other & 3 & 3 & 100.0 & \\
\hline \multirow{4}{*}{ FrequencyofBrushing } & Morning only & 309 & 295 & 95.4 & \multirow{4}{*}{$\mathrm{p}=0.012(\mathrm{sig})$} \\
\hline & Night only & 11 & 10 & 90.9 & \\
\hline & Morning and Night & 122 & 101 & 82.7 & \\
\hline & After every meal & 4 & 3 & 75.0 & \\
\hline \multirow{3}{*}{ Tooth brushing assisted/supervised } & Parent alone & 65 & 56 & 86.1 & \multirow{3}{*}{$\mathrm{p}=.006(\mathrm{hs})$} \\
\hline & Child under parental supervision & 56 & 50 & 89.2 & \\
\hline & Child alone & 306 & 293 & 95.7 & \\
\hline \multirow{2}{*}{ Rinsing mouth after food } & Yes & 251 & 224 & 89.2 & \multirow{2}{*}{$\mathrm{p}=0.739$ (ns) } \\
\hline & No & 249 & 239 & 95.9 & \\
\hline
\end{tabular}

Table 4. Dietary habits and ECC prevalence.

\begin{tabular}{|c|c|c|c|c|c|}
\hline \multirow{2}{*}{ Dietary habits } & \multirow{2}{*}{ Variables } & \multicolumn{4}{|c|}{ Tibetan } \\
\hline & & $\mathbf{N}$ & $\mathbf{n}$ & $\%$ & $\mathbf{P}$ \\
\hline \multirow{2}{*}{ Breast Feeding } & Yes & 451 & 363 & 80.4 & \multirow{2}{*}{$0.349 \mathrm{~ns}$} \\
\hline & No & 49 & 24 & 48.9 & \\
\hline \multirow{2}{*}{ Bottle Feeding } & Yes & 407 & 376 & 92.3 & \multirow{2}{*}{$<0.001$ vhs } \\
\hline & No & 93 & 41 & 44.0 & \\
\hline \multirow{2}{*}{ Sugar added to the bottle } & Yes & 326 & 277 & 84.9 & \multirow{2}{*}{$<0.001 \mathrm{vhs}$} \\
\hline & No & 81 & 81 & 100.0 & \\
\hline \multirow{2}{*}{$\begin{array}{l}\text { Sugar added to the other food } \\
\text { (table sugar/jagery/honey) }\end{array}$} & Yes & 338 & 335 & 99.1 & \multirow{2}{*}{$<0.001$ vhs } \\
\hline & No & 46 & 38 & 82.6 & \\
\hline \multirow{3}{*}{ In-between-meal snacking } & Yes & 94 & 80 & 85.5 & \multirow{3}{*}{$<0.001$ vhs } \\
\hline & No & 304 & 123 & 40.4 & \\
\hline & NotAnswered & 102 & 96 & 94.1 & \\
\hline \multirow[t]{2}{*}{ sweets intake(chocolates, candies,toffes) } & No & 73 & 14 & 19.1 & \multirow[t]{2}{*}{$<0.001$ vhs } \\
\hline & NotAnswered & 17 & 17 & 100.0 & \\
\hline
\end{tabular}


Table 5. Dental visits and ECC prevalence.

\begin{tabular}{|c|c|c|c|c|c|}
\hline \multirow{2}{*}{ Population } & & \multicolumn{4}{|l|}{ Tibetan } \\
\hline & & $\mathbf{N}$ & $\mathbf{n}$ & $\%$ & $\mathbf{P}$ \\
\hline \multirow{2}{*}{ Dental visits } & Yes & $85(17 \%)$ & 85 & 100.0 & 0.006 \\
\hline & No & $415(83 \%)$ & 378 & 91.0 & Hs \\
\hline
\end{tabular}

\section{Acknowledgements}

The authors wish to extend their gratitude to all the parents, school teachers and marvelous children for being cooperative and assisting in this study. We would especially like to acknowledge Mr.Tamdin Tsering Janyeal, Director, Tibetan SOS children's Village, the Representatives of Lugsom Samdupling Tibetan Settlement, Tibetan Dickey Larsoe settlement and Rabgayling Settlement Bailakuppe, Mysore District; India for giving the permission to conduct this study.

\section{References}

[1] American academy of pediatric dentistry (AAPD): policy on early childhood caries: unique challenges and treatment options (2003): Pediatrc Dent special issue 24(7),24-25.

[2] Cariono, KM., Shinada, K. and Kawaguchi, Y. (2003): Early childhood caries in northern Philippines. Community Dent Oral Epidemiology31, 81-89.

[3] Chan, SCL., Tsai, JSJ. and King, NM. (2002): Feeding and oral hygiene of preschool children in Hong Kong and their caregivers' dental knowledge and attitudes. Int J. of Paediatric Dentistry12, 322-331.

[4] Clemencia, M., Vargas, CM., Manajemy, N., Pooja, K. and Tinanoff, N. (2000): Oral health status of preschool children attending head start in Maryland. Pediatric Dentistry24(3),257-263.

[5] Cunnigham, SED., Farokhi, M., Baez, M. and Cano, S. (2008): Prevalence of early childhood caries in young Mexican American children. IADR $86^{\text {th }}$ General session and exhibition $3^{\text {rd }}$ July 2008, Caries research - epidemiology.

[6] Dominguez, RV., Astasio, AP. andOrtega, MP. (1993): Analysis of several risk factors involved in dental caries through multiple logistic regressions. Int Dent J43, 149-156.

[7] Ekman, A., Holm, AK., Schelin, B. and Gustafson, L. (1981): Dental health and parental attitudes in Finnish immigrant preschool children in the north of Sweden. Community Dent Oral Epidemiol9(5),224-229.

[8] Filstrup, SL., Briskie, D., Da-Fonseca, M., Lawrence, L., Wandera, A. and Inglehart, MR. (2003): Early childhood caries and quality of life: child and parent perspectives. Pediatr Dent 25, 431-440.

[9] Jin, BH., Ma, DS., Moon, HS., Paik, DMI., Hahn, SH. And Horowitz, AM. (2003): ECC prevalence and risk factors in Seoul, Korea. American Assoc Pub Health Dentistry23, 183188

[10] Jose, B. and King, NM. (2003): Early childhood caries lesions in preschool children in Kerala, India. Pediatr Dent 25(6),594600 .
[11] Kaste, LM.,Marianos, D., Chang, R. and Phipps, KR. (1992): The assessment of nursing caries and its relationship to high caries in the permanent dentition. J Public Health Dent 52, 64-68.

[12] Kuriakose, S. and Joseph, E. (1999): Caries prevalence and its relation to socioeconomic status and oral hygiene practices in 600 pre-school children of Kerala-India. J Int Soc Prev Ped Dentistry17(3), 97-100.

[13] Laws, MB. (2001): Race and ethnicity in biomedical and health services research. Archives of Pediatrics and Adolescent Medicine155, 972-974.

[14] Li, Y. and Wang, W. (2002): Predicting caries in permanent teeth from caries in primary teeth: an eight-year cohort study. J Dent Res81, 561-566.

[15] Low, W., Tan, S. and Schwartz, S. (1999): The effect of severe caries on the quality of life in young children. Pediatr Dent 21 , 325-326.

[16] Man Qin., Jing Li., Sun Zhang. And Wenli Ma. (2008): Risk factors for severe ECC in children younger than 4 yrs old in Beijing; China. Pediatric Dentistry30(2), 122-128.

[17] Man Wai Ng. (2003): Multicultural influences on child-rearing practices: implications for today's pediatric dentist. Pediatric Dentistry 25(1),19-22.

[18] Murray, JJ. (1996): Prevention of Oral Disease. 3rd Edition,.pp 3\&231.

[19] Namal, N., Vehit, HE. and Can, G. (2005): Risk factors for dental caries in Turkish pre-school children. $J$ Indian Soc Pedod Prev Dent23(3),115-118.

[20] Poulsen, S. and Scheutz, F. (1999): Dental caries in Danish children and adolescents 1988-1997. Community Dent Health16, 166-170.

[21] Robert, J. and Berkowitz. (2003): Causes, treatment and prevention of ECC: A Microbiological Perspective. J.Can Dent Assoc69(5),304.

[22] Robert, JS., Douglas, JB., Michael, EK. and Moffatt. (2007): Caregiver knowledge and attitudes of preschool oral health and early childhood caries (ECC). International Journal of Circumpolar Health66(2),153-167.

[23] Robert, JS., Pamela, JS. and Jeanne, CW. (2005): Prevalence of caries among preschool-aged children in a northern Manitoba Community. JCDA71(1),567.

[24] Robertson, J.,McL, A., Reade, PC., Steidler, NE. and Spencer, AJ. (1989): A dental survey of Tibetan children in Dharamsala. Community Dent Oral Epidemiol17, 44-46.Rodrigues, CS. and Sheham, A. (2000): The relationship between dietary guidlines, sugar intake and caries in primary teeth in low income Brazilian 3 year olds: a longitudinal study. Int J. of Paediatric Dentistry10, 47-55. 
[25] Sweeney, PC. and Gel bier, S. (1999): The dental health of pre-school children in a deprived urban community in Glasgow. Community Dent Health16, 22-25.

[26] Tang, JMW., Altman, DS., Robertson, DC., O'Sullivan, DM., Douglass, JM. And Tinanoff, N. (1997): Dental caries prevalence and treatment levels in Arizona pre-school children. Public Health Reports112, 319-329.

[27] Tracy, L., Finlayson., Kristine, S., Ismail., Amid, I. and Woosung, S. (2007): Psychosocial factors and early childhood caries among low-income African-American children in Detroit. Community Dent Oral Epidemiol35, 439-448.

[28] Vadiakas, G. (2008): Case definition, etiology and risk assessment of early childhood caries (ECC): A revisited review. European Archives of Pediatric Dentistry9(3),114-125.
[29] Valaitis, R., Hesch, R., Passarelli, C., Sheehan, D. and Sinton, J. (2000): A systemic review of relationship between breastfeeding and ECC. CanadianJ of Pub Health 91(6),411417.

[30] Verrips, GH.,Kalsbeek, H. and Eijikman, MA. (1993): Ethnicity and maternal education as risk indicators for dental caries and role of dental behavior. Community Dent Oral Epidemiol21, 209-214.

[31] Vivek, DK. (2010): Early Childhood Caries-An Insight. $J$ Int Oral health2(1), 1-9. 